\title{
What is the Neurodivergent Aesthetic?
}

\author{
Kazimir Bielecki \\ Artist \\ DYSPLA, 12, TGEC, Town Hall \\ Approach Road, London, N15 4RX, UK \\ kaz@bieleckiandbielecka.com
}

\author{
Lennie Varvarides \\ Artist \\ DYSPLA, 12, TGEC, Town Hall \\ Approach Road, London, N15 4RX, UK \\ hello@dyspla.com
}

\section{INTRODUCTION}

\begin{abstract}
Neurodivergent: neurocognitive functioning that diverges significantly from the dominant societal standards of 'normal', including dyslexics, dyspraxics, $A D(H) D$, autistics, dyscalculics, OCD, dysgraphics, Tourettes, etc. (Hewlett \& Cooper 2017).
\end{abstract}

When neurodivergence is mentioned in some parts of society it is generally met with "negative thoughts and attributions" (Armstrong 2010), in other parts of society there is talk of cures and "with the increasing threat that genetic engineering and prenatal screening pose in potentially eliminating neurodiverse people from the planet" (Armstrong 2010). Moving image artists such as Steve McQueen, David Lean, Charlie Chaplin, Stan Brakhage, Stanley Kubrick, Steven Spielberg, Quentin Tarantino, Martin Scorsese and Alejandro G. Iñárritu are some of the most well known moving image-makers who are identified as neurodivergent.

Primarily using phenomenological and ethnographic research methods we will look at the traits that define neurodivergence and evaluate whether the working practices of neurodivergent moving image-makers, can be attributed to the definition of the Neurodivergent Aesthetic (Shaughnessy 2013). What cognitive disabilities/advantages do Moving Image Artists exhibit when working and does this neurodivergent methodology imprint on our screens? This is the initial stages of research and will continue over the next few years, culminating in a series of filmic portraiture and installations exhibiting the methods described within the research. By exploring the neurodivergent aesthetic we hope to highlight the creative advantages of neurodivergence in the hope to nurture our current and future talents within the UK creative industry for cultural and economic benefit.

\section{NEURODIVERGENT CREATIVE TRAITS}

There is a shame that clouds the colourful plethora of neurodivergent minds within the creative industry and many still see neurodivergence as a disability and a hindrance to industry structures. Neurodivergent traits such as: spatial, visual, big picture thinking and dynamic reasoning (Edie \& Edie 2011) hyperfocus (Carnes \& Holloway 2009); narrative reasoning and strength of episodic memory (Edie \& Edie 2011); spontaneity and creative cognition (White 2018); and strengths in creative intuition (LaFrance 1997), can be attributed to the successful production of moving image. Each trait is an essential aspect of moving image making practice. The ability to see beyond what's ahead and around the corner is utilised through spatial and big picture thinking. Explained in the Dyslexic Advantage by Dr Brock Edie and Fernette Edie (2011) as M-Strengths "to create a continuous, interconnected series of 3-D perspectives". An artist's relationship with their life experiences is expressed through episodic memory. Termed 'narrative reasoning' it has "a highly narrative or "scene-based" format in which concepts and ideas are conceived or recalled as experiences, examples or enactments" (Edie \& Edie 2011). Creative Intuition and Dynamic Reasoning is described as "the ability to 'read' patterns in the real world that allow us to reconstruct past events we haven't witnessed, predict likely future events or simulate and preview plausible outcomes of interventions or various courses of action" (Edie \& Edie 2011).

\section{ND ARTISTS \& NEURODIVERGENT METHODOLOGY}

In the case of Award winning neurodivergent filmmaker Mike Forshaw, the initiation of his ideas stems from strong moments in his memory, 
analysing the emotional detail and following intuitive forms of narrative structure:

\begin{abstract}
"In film, things don't have to be linear. I feel it is important to draw an audience into your story in the same way you were initially drawn to the idea. People have difficulty with that sometimes because in a conventional narrative that idea should obey a certain structure or rule. But to me, if it feels right to you as the filmmaker, then you should always break the convention and trust the feeling" (Forshaw 2018).
\end{abstract}

Neurodivergent moving image-makers will create intuitive environments where they can allow themselves and their team to thrive. Shia LaBeouf, a neurodivergent actor working with neurodivergent director Michael Bay, while on the set of the film Transformers says, Bay:

"[P]uts you as close as you can get to the flames - or as close to the helicopter explosion without charring or smashing your life. When you're hanging off a rooftop and you feel like you're going to die, you don't want the auteur director who's going to say, "Let's go through your emotional history or discuss mannerisms." In that sense, he is an actor's director - he doesn't want you to have to conjure it. He wants you to be experiencing it." (Saroyan 2007).

\section{THE NEURODIVERGENT AESTHETIC?}

Neurodivergent Video Artist and world renowned VJ D-FUSE's (a.k.a. Mike Falkner) believes his work is lead by his intuitive process, a synesthetic understanding of shapes, colours and sounds.

\begin{abstract}
"The neurodivergent aesthetic is an intuitive process that works for the art, as opposed to a defining style. I like building irregular textures, sounds and images into 'irregular regular' patterned structures. I see it as a normal work process but others see it as quite strange. I build an intuitive sense of rhythm and process to the way I chop and make things. Creating non regular patterers, sounds or images becomes more human, more intuitive." (Falkner 2018)
\end{abstract}

What is clear is that intuition seems to be a key creative tool for most neurodivergent moving image-makers but their methodology is often unwelcome within industry structures. It is no secret that the creative industries are populated with high numbers of neurodivergent people, and without the necessary upbringing and structures conducive to insightful thought (Edie \& Edie 2011), many great minds are being lost. By exploring the neurodivergent aesthetic we hope to highlight the importance to address the diversity disparity within the creative Industry (Bhavnani 2007). We must reevaluate the rigid structures that populate the creative industries to allow intuition and innovation to thrive. The education system, like our society as a whole, still undervalues the importance of neurodivergence and of learning and thinking holistically (Cooper 2011). Following the social model of disability and looking at the creative advantages of neurodivergence, if we create an environment where neurodivergent practitioners can flourish and their natural abilities are nurtured (Petersen 2012) could this advantage the industry creatively and economically?

\section{REFERENCES}

Armstrong, T. (2011) The Power of Neurodiversity: Unleashing the Advantages of Your Differently Wired Brain. Da Capo Lifelong Books, Boston.

Bhavnani, R. (2007) Barriers to Diversity in Film. https://www.bfi.org.uk/sites/bfi.org.uk/files/downloads/ukfilm-council-barriers-to-diversity-in-film-2007-08-20.pdf (retrieved 1 April 2019).

Cooper, R. (2011) Neurodiversity and Dyslexia: Compensatory strategies, or different approaches? https://www.achieveability.org.uk/files/1460064671/neuro diversity-and-dyslexia-by-ross-cooper.pdf (retrieved 1 April 2019).

Eide, B. and Eide, F. (2011) The Dyslexic Advantage: Unlocking the Hidden Potential of the Dyslexic Brain. Hay House, London.

Hewlett, K. and Cooper, R. (2018) The Westminster AchievAbility Commission for Dyslexia and Neurodivergence 2018. AchieveAbility, London.

LaFrance, E. (1997) The gifted/dyslexic child: Characterizing and addressing strengths and weaknesses.

https://link.springer.com/article/10.1007/s11881-9970025-7 (retrieved 1 April 2019).

Petersen, S. (2019) Dyslexia and Creativity. https://www.huffingtonpost.com/soren-petersen/dyslexiaand-creativity b 1531298.html (retrieved 1 April 2019).

Sacks, O. (1998) Oliver Sacks inaugural lecture for the Centre for the mind Canberra Australia, 10th January 1998.

https://www.abc.net.au/radionational/programs/sciencesh ow/dr-oliver-sacks-transcript-attached/3564542 (retrieved 1 April 2019).

Saroyan, S. (2007) The incredible exploding man. https://www.telegraph.co.uk/culture/3666728/Theincredible-exploding-man.html (retrieved 1 April 2019).

Shaughnessy, N. (2013) Imagining Otherwise: Autism, Neuroaesthetics and Contemporary Performance. https://www.tandfonline.com/doi/abs/10.1179/030801881 3Z.00000000062?journalCode=yisr20 (retrieved 1 April 2019).

White, H. (2018) Thinking "Outside the Box": Unconstrained Creative Generation in Adults with Attention Deficit Hyperactivity Disorder. https://onlinelibrary.wiley.com/doi/10.1002/jocb.382 (retrieved 1 April 2019). 